\title{
Editorial for QShine 2008
}

\author{
Guest Editors QShine 2008
}

Published online: 23 January 2009

(C) Springer Science + Business Media, LLC 2009

Recent technological developments in wireless communications and mobile networks, broadband networks, pervasive computing, and distributed computing have led to a wide variety of new challenging problems. These include the provisioning of Quality of Service (QoS), survivability, resilience and scalability in a wide range of emerging applications and services over large-scale wired and wireless networks. The Fifth International Conference on Heterogeneous Networking for Quality, Reliability, Security and Robustness (QShine 2008) was held in Hong Kong in July 2008, which focuses on the research challenges associated the design and implementation of large-scale wired and wireless networks and distributed systems. The aim of this conference is to bring together research and practitioners to present and share the latest results in the areas of performance, configuration, cross-layer approaches, scalability, resilience and survivability of large scale heterogeneous networks and distributed systems.

More than 100 papers have been received in this conference, out of which we have selected 13 high quality papers for this special issue. This special issue covers the topics of Quality of Service provisioning and resource management, QoS in WLAN, WPAN, and WiMAX, wireless ad hoc networks, wireless mesh networks, wireless sensor networks, cross-layer performance optimization, and security protocols in wireless networks. Besides the above traditional topics, some new and emerging research directions, such as cognitive radio networks, have also been included in this special issue.

This special issue starts with the paper "Harnessing Traffic Uncertainties in Wireless Mesh Networks - A Stochastic Optimization Approach" by Yang, Chi, and Yuguang. The authors investigated the routing optimization problem in wireless mesh networks. Considering the actual traffic is time-varying and difficult to measure, in this paper, a stochastic optimization framework was pursued where the expected network utility is maximized.

Tehuang, Wanjiun, and Jeng-Farn in "Distributed Contention-Aware Call Admission Control for IEEE 802.11 Multi-Radio Multi-Rate Multi-Channel Wireless Mesh Networks" studied call admission control (CAC) in IEEE 802.11 multi-radio multi-rate multi-channel (MR2-MC) wireless mesh networks (WMNs). A CAC mechanism, called Contention-Aware Multi-channel Call Admission Control, has been proposed.

Yuxia, Joo-Han, and Vincent in "Cooperative Protocols Design for Wireless Ad-Hoc Networks with Multi-hop Routing" proposed a complete system framework for wireless ad-hoc networks utilizing two different cooperative relaying techniques at the physical layer: the repetition coding and the space-time coding.

Hany, Weihua and Bruno in "DTN Based Dominating Set Routing for MANET in Heterogeneous Wireless Networking" proposed a super node system architecture to achieve the connectivity over interconnected heterogeneous wireless access networks, which employs the delay-tolerant network (DTN) concept to overcome the problem of potential intermittent connections caused by user roaming and ensures message delivery in the presence of a long disconnection period.

Hui, Jiannong, and Xiaopeng in "GMZRP: Geographyaided Multicast Zone Routing Protocol in Mobile Ad Hoc Networks" presented the design and evaluation of a highly efficient on-demand multicast routing protocol for MANETs. The protocol, called Geography-aided Multicast Zone Routing Protocol (GMZRP), eliminated as much as possible duplicate route queries by using a simple yet effective strategy for propagating the multicast route request (MRREQ) packets. 
Chengqi and Qian in "OMH - Suppressing Selfish Behavior in Ad hoc Networks with One More Hop" proposed One More Hop (OMH) protocol which suppresses selfish behavior from a totally new angle. Basing on the fact that the selfish but rational nodes still want to receive and send packets, if a node cannot determine whether a packet is destined for it or not, it cannot drop the packet. With modified routing protocol and cryptographic techniques, $\mathrm{OMH}$ achieves this design target.

Yanchao and Kui in "On Address Privacy in Mobile Ad Hoc Networks" identified address privacy as a new security requirement to prevent attackers from ascertaining network addresses of MANET principals. They further presented Swarms, the first solution to satisfying this requirement.

Yuan and Danny in "Joint Rate-and-Power Allocation for Multi-channel Spectrum Sharing Networks" studied the joint rate-and-power allocation for multi-channel spectrum sharing networks. They formulated this cross-layer optimization problem as a non-cooperative game GJRPA in which each user has a coupled two-tuple strategy, i.e., simultaneous rate and multi-channel power allocations. A multi-objective cost function was proposed to represent user's awareness of both QoS provisioning and power saving.

Lin, Lin, Xuemin, and Jon in "Resource Management and QoS Provisioning for IPTV over mmWave-based WPANs with Directional Antenna" proposed a method to efficiently support high definition video flows in a mmWave-based WPAN with QoS guarantee, considering the characteristics of both the IPTV traffic and the mmWave communication technology.

Min, Victor, and Shiwen in "Directional Controlled Fusion in Wireless Sensor Networks" proposed the notation of "multipath expanding" for data aggregation in wireless sensor network to solve the multiple flows competing problem by jointly considering data fusion and load balancing. A novel directional-controlled fusion (DCF) scheme is proposed, which consists of two key algorithms termed as directional control and multipath fusion. By adjusting multipath fusion factor in DCF, the trade-offs between multipath-converging and multipath-expanding can be easily achieved.

Yueming, Qing, Fangming, Gabriel, and Bo in "The Sink Node Placement and Performance Implication in Mobile Sensor Networks" presented a mobile sensor network architecture consisting of a potentially large number of mobile sensors and a single or multiple stationary sink nodes for sensing information collection. They formulated a distinct coverage measurement problem in term of sensing information collection and studied the relevant performance and examined the effect from a variety of relevant factors.

Yangfan, Michael, and Jiangchuan in "On Sensor Network Reconfiguration for Downtime-Free System Migration" presented the first formal study on the problem of downtime-free migration. They demonstrated that the downtime can effectively be eliminated, by partitioning the sensors into subsets, and let them perform migration tasks successively with the rest still performing normal services.

Chunwang, Zhen, Yawen, Steve, and Yong in "A Statistical Indoor Localization Method for Supporting Location-based Access Control" proposed a statistical indoor localization method. In an offline phase, the authors fitted a locally weighted regression and smoothing scatterplots model on the signal strength received at different training locations, and built a radio map that contains the distribution of signal strength. In an online phase, they determined the locations of unknown points using maximum likelihood estimation based on the measured signal strength and the stored distribution.

Before closing this editorial, we would like to thank those who contributed significantly behind the scene towards the success of this special issue. Special thanks to Dr. Imrich Chlamtac, Prof. Yuguang (Michael) Fang and Prof. Xuemin (Sherman) Shen for their advice and encouragement during the process of putting together this Special Issue. We hope that you will enjoy reading this Special Issue.

\section{Qian Zhang and Ekram Hossain}

Qian Zhang (M'00-SM'04) received the B.S., M.S., and Ph. D. degrees from Wuhan University, China, in 1994, 1996, and 1999, respectively, all in computer science.

Dr. Zhang joined Hong Kong University of Science and Technology in Sept. 2005 as an Associate Professor. Before that, she was in Microsoft Research, Asia, Beijing, China, from July 1999, where she was the research manager of the Wireless and Networking Group. Dr. Zhang has published more than 200 refereed papers in international leading journals and key conferences in the areas of wireless/ Internet multimedia networking, wireless communications and networking, and overlay networking. She is the inventor of about 30 pending patents.

Dr. Zhang is the Associate Editor for IEEE Transactions on Wireless Communications, IEEE Transactions on Multimedia, IEEE Transactions on Vehicular Technologies (2004-2008), Computer Networks and Computer Communications. She also served as Guest Editor for IEEE JSAC, IEEE Wireless Communications, Computer Networks, and ACM/Springer MONET. Dr. Zhang has been involved in organization committee for many important ACM and IEEE conferences, including MobiCom, MobiHoc, Infocom, ICC, Globecom, WCNC, etc. Dr. Zhang has received TR 100 (MIT Technology Review) world's top young innovator award. She also received the Best Asia Pacific (AP) Young Researcher Award elected by IEEE Communication Society in year 2004. She received the Best Paper Award in 
Multimedia Technical Committee (MMTC) of IEEE Communication Society and Best Paper Award in QShine 2006, Globecom 2007, and ICDCS 2008. She received the Oversea Young Investigator Award from the National Natural Science Foundation of China (NSFC) in 2006.

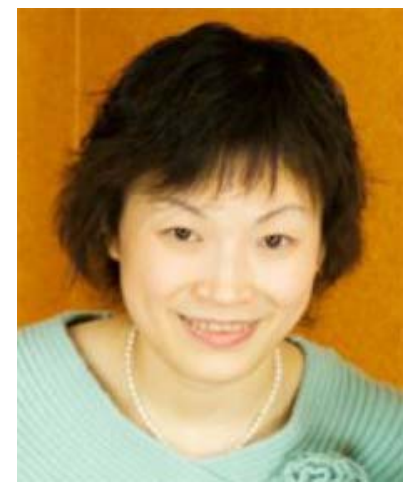

Ekram Hossain ( $\left.\mathrm{S}^{\prime} 98-\mathrm{M}^{\prime} 01-\mathrm{SM}^{\prime} 06\right)$ is currently an Associate Professor in the Department of Electrical and Computer Engineering at University of Manitoba, Winnipeg, Canada. He received his Ph.D. in Electrical Engineering from University of Victoria, Canada, in 2000. His research interests include modeling, analysis, and optimization of wireless communication networks and cognitive radio systems. He is a co-author/co-editor/editor of the books Dynamic Spectrum Access and Management in Cognitive Radio Networks (Cambridge University Press, 2009), Wireless Mesh Networks: Architectures and Protocols (Springer, 2007), Heterogeneous Wireless Access networks (Springer, 2008), Introduction to Network Simulator NS2 (Springer, 2008), and Cognitive Wireless Communication Networks (Springer,
2007). Dr. Hossain serves as an editor for the IEEE Transactions on Mobile Computing, the IEEE Transactions on Wireless Communications\}, the IEEE Transactions on Vehicular Technology, IEEE Wireless Communications, and several other international journals. He served as a guest editor for the special issues of IEEE Communications Magazine on Cross-Layer Protocol Engineering for Wireless Mobile Networks and Advances in Mobile Multimedia, and IEEE Wireless Communications on Radio Resource Management and Protocol Engineering for IEEE 802.16. Dr. Hossain served as a technical program co-chair for IEEE Globecom' 07, IEEE WCNC'08, IEEE VTC'08 (Fall), and QShine'08. Also, he served as Technical Program Chair for the Workshop on Cognitive Wireless Networks (CWNets '07) and the Workshop on Wireless Networks for Intelligent Transportation Systems (WiNITS '07) held in conjunction with QShine'07, August 14-17, Vancouver, Canada. Dr. Hossain is a registered Professional Engineer (P.Eng.) in the province of Manitoba, Canada.

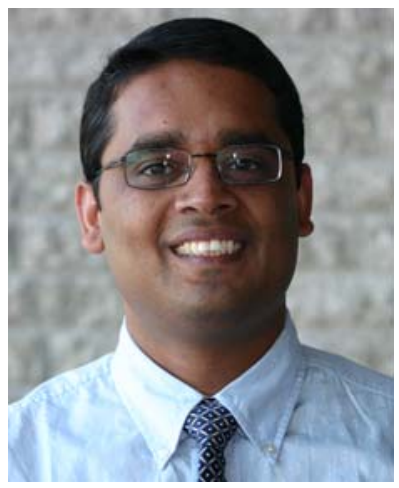

